\title{
Erratum to: Lax-Friedrichs Multigrid Fast Sweeping Methods for Steady State Problems for Hyperbolic Conservation Laws
}

\author{
Weitao Chen ${ }^{1}$ - Ching-Shan $\mathrm{Chou}^{2}$ - Chiu-Yen Kao ${ }^{3}$
}

Published online: 12 April 2015

(C) Springer Science+Business Media New York 2015

\section{Erratum to: J Sci Comput DOI 10.1007/s10915-015-0006-7}

The authors would like to correct reference 31 published in the original version as follows:

Engquist, B., Froese, B.D., Tsai, Y.-H.R.: Fast sweeping methods for hyperbolic systems of conservation laws at steady state. J. Comput. Phys. 255, 316-338 (2013).

The online version of the original article can be found under doi:10.1007/s10915-015-0006-7.

$凶$ Ching-Shan Chou

chou@math.ohio-state.edu

Weitao Chen

weitaoc1@uci.edu

Chiu-Yen Kao

Ckao@cmc.edu

1 Department of Mathematics, University of California, Irvine, CA 92617, USA

2 Department of Mathematics, The Ohio State University, Columbus, OH 43210, USA

3 Department of Mathematical Sciences, Claremont McKenna College, Claremont, CA 91711, USA 